\title{
La Función de Ser Padres y Madres, Vida Cotidiana y Retos Actuales. Entre la prevención y la asistencia, la intervención en el ámbito de los malestares cotidianos
}

\section{The Role of Fathers and Mothers, Everyday Life and Current Challenges. Prevention, Care and Intervention in Day-to-Day Uneasiness}

\author{
Mirtha Cucco García \\ Ambito privado, España
}

\begin{abstract}
Resumen. Favorecer el desarrollo de la autonomía en niños, niñas y adolescentes es un reto social tanto de las familias, como de los diversos profesionales y ámbitos institucionales. La autonomía se construye en un proceso en el que influyen el ámbito familiar, el escolar y todos los espacios de inserción social. Pero en el marco de las contradicciones sociales actuales, los roles asignados a hombres y mujeres, la dificultad de conciliación de espacios familiares y laborales, el tiempo y las prisas, el consumismo y el abuso telemático junto al desprestigio de los aprendizajes, la indigencia de futuro, entre otras cuestiones, generan grandes dificultades para llevar a cabo esta tarea.

Nuestra reflexión se encuadra desde la concepción de la Metodología ProCC. Esta sostiene: la construcción socio-histórica de la subjetividad; la interpelación de paradigmas desde el intento de superar disociaciones paralizantes entre lo social y lo individual; la necesidad de recuperación de capacidad instituyente y la necesidad de generar espacios grupales de reflexión que permitan interpelar aquello que siendo normalizado, invisibiliza lo saludable con grandes costos en salud y bienestar.
\end{abstract}

Palabras clave: vida cotidiana, familia, crianza, autonomía, grupo.

\begin{abstract}
Promoting the development of independence among children and teenagers is a social challenge for both the families and the different professionals and institutions involved. Becoming independent is a process influenced by the family, the school and the
\end{abstract}

Dra. en Psicología por la Universidad Complutense de Madrid. Especialista en Psicología Social. Titulo de Psicóloga Especialista en Psicología Clínica. Directora de la Casa Central del Centro de Desarrollo en Salud Comunitaria “Marie Langer”, en Madrid. Coordinadora del Dpto. Docente nacional e internacional de la Especialidad en Metodología ProCC.

Autora de la Metodología de los Procesos Correctores Comunitarios y de sus Programas de Intervención comunitaria tales como Escuela para Padres, El rol de la mujer, problemática actual; El rol del hombre, una problemática silenciada; La problemática hombre-mujer, encuentros y desencuentros; Ser joven hoy; Adolescencia, ¿Asignatura pendiente?, Taller de juego; El adulto mayor y su lugar vital-social; Vivir el cuerpo; Intervención en el ámbito escolar; El rol del profesor, aprendizaje, grupo y conflictos relacionales en el aula; entre otros.

La correspondencia sobre este artículo debe enviarse al E-mail: mirtha@procc.org 
educational system, and together with the spaces of social insertion. However, within the framework of today's social contradictions, such as the roles assigned to men and women; the difficulties to reconcile family and work spaces; the time pressure and rush; consumerism and the the telematical devices, plus, amongst other issues, the fact that learning is in itself undervalved together with the poor prospects for the future give rise to great difficulties when trying to accomplish this task.

Our approach is based on the idea proposed by the ProCC which means: the social-historical construction of subjectivity; the questioning of paradigms taking roots in the intention to overcome the paralysing effects caused by dissociation between the individual and society; the need to recover our capacity to transform and the need to generate spaces for group debate that allows us to question matters that, wjile in the process of being normalized, are at the same time hiding whatever is health damages for the well-being.

Keywords: daily life, family, growth, breeding, autonomy, independent, group.

\section{Introducción}

"Atender las necesidades de salud de la población en el marco de las contradicciones sociales actuales, plantea a nuestras ciencias y a nuestra praxis, un importante desafío ideológico, ético y de compromiso social, así como teórico y metodológico. Las acciones en salud no pueden disociarse de las condiciones de vida de las gentes, por tanto atenderlas de forma integral implica partir de un buen análisis de la realidad y contar con el desarrollo del protagonismo y la acción participativa de la población como elemento central para la articulación de respuestas y soluciones"

(Сисco, 2009, p.1).

Vida cotidiana hoy... hombres y mujeres “sin tiempo”, padres y madres preocupados, maestro/as desconcertados, desgaste profesional, niño/as con trastornos de concentración, fracaso escolar, jóvenes indigentes de futuro, desencuentro de hombres y mujeres, la pérdida de sentido, la banalización de las normas, la inmediatez...

Nos encontramos hoy cada vez más con la propuesta de un sujeto torpemente vivo, con fragilidad narcisista, inhábil en el reconocimiento del otro, en el manejo de los conflictos y en el sostén de un vínculo, con predominio de defensas primitivas y gran sentimiento de vacío o de pérdida de sentido.

Nos enfrentamos, en este sentido, a retos que requieren una atención urgente y priorizada. En el contexto de las contradicciones sociales actuales observamos un impacto devastador en relación al deterioro de la vida cotidiana y la precarización subjetiva. Asistimos a los mayores niveles conocidos de fragmentación social, con el extrañamiento del sujeto y la suplantación de redes socio-afectivas por redes cibernéticas.

Los procesos de un crecer saludable se ven seriamente afectados. La apología del cumplimiento inmediato de deseos, de la baja tolerancia a la frustración, junto al desprestigio de las normas y la dificultad adulta de poner límites adecuados, la falta de esfuerzo y la inmediatez que niega la idea de proceso, dificultan los procesos de aprendizaje. Cada vez más encontramos niños y niñas diagnosticados de déficit atencional, descontrol de impulsos y agresividad, junto a dosis de sobreestimulación de cosas que no pueden procesar, y promesas de abastecimiento absoluto. La capacidad de interreaccionar suplanta a la capacidad de interrelacionarse. Los chicos de hoy "hablan mucho, escuchan poco y piensan nada", expresaba con preocupación un maestro.

La construcción del narcisismo, la elaboración de los duelos y los procesos de triangulación como "tres grandes organizadores de la subjetividad" (Cucco, 2003, p.81), se encuentran en una extrema precarización. Se trata de un sujeto que adolece de un narcisismo generoso, de la capacidad de elaborar duelos, de la capaci- 
dad de sostener los ejes de la identidad-diferencia, del deseo-prohibición y de la relación yo-alteridad. Sujetos entonces, con dificultades en la capacidad de sostener el deseo, una temporalidad que trascienda un presente continuo y la posibilidad de articulación de sus proyectos vitales.

Los modelos de identificación sexual están atravesados por la ambigüedad y la dificultad con las diferencias desde los paradigmas de la lógica binaria, atributiva y jerárquica; las relaciones hombre-mujer están marcadas por el desencuentro, la confusión, la violencia en muchos casos; hay cada vez menos espacio para la convivencia y el encuentro generoso, y observamos muchas soledades "autoabastecidas". Entendemos que estos grandes macroindicadores sociales actuales se materializan y guardan una relación directa con las pautas de crianza hegemónicas y afectan severamente la función de ser padres y madres.

¿Qué hacer cuando esta situación es cada vez más masiva e invisibilizada, constituyendo lo que hemos dado en llamar el ámbito de la Normalidad Supuesta Salud? ¿Qué hacer cuando esto nos enfrenta al obstáculo epistemofílico de estar construidos nosotros mismos con aquellas categorías que queremos transformar? ¿Qué métodos que no impliquen una propuesta de psiquiatrización de la población pudieran ser eficaces? ¿Cómo diferenciarlos de los de las acciones de prevención o de los de los espacios terapéuticos?¿Qué interpelación paradigmática hemos de hacer para que las teorías arrojen luz para una intervención eficaz de estos malestares? ¿Qué lugar ocupa lo grupal y comunitario?

\section{La Normalidad Supuesta Salud}

Nos enfrentamos entonces a malestares comunes, malestares de la vida cotidiana actual, que formando parte de los consensos sociales instituidos quedan invisibilizados tras su status de "normales" y sólo se visibilizan algunos de sus efectos, a los que se suele atender disociados de sus causas. Esto conlleva la situación paradojal de que las intervenciones, disminuyendo el dolor de un efecto, reafirma sus causas. Esto explica en parte los niveles de impotencia de los profesionales y la desilusión y la desgana, cuando no el síndrome del profesional quemado.

Desde la Metodología de los ProCC, acuñamos el concepto de Normalidad Supuesta Salud para referirnos a estos malestares, y los definimos como (Cucco, 2006a, p.32):

"Aquellos que la gente sufre y habitualmente no analiza ni cuestiona porque los considera normales. Aquellos que no generan demanda explícita, no tienen interlocutor válido, engrosan la cultura de la queja y para los cuales no existe un campo de intervención específico; brindándose las respuestas habituales desde enfoques terapéuticos-asistenciales que, o bien tienden a medicalizarlos, psiquiatrizarlos o categorizarlos como pertenecientes a grupos de riesgo social; o bien a incluirlos en acciones preventivas inespecíficas, quedando la mayor parte de las veces en tierra de nadie”.

La sistematización de estos malestares constituyen los Indicadores Diagnósticos de Población, que poseen tanto un carácter diagnóstico respecto de las necesidades de las gentes, como también pronóstico en relación a la mayor o menor resistencia al cambio en los procesos de intervención sobre los mismos.

Para comprender la génesis de estos malestares, es necesario focalizar la mirada en ese justo punto de cruce que representa el lugar de encuentro entre lo más íntimo personal, con elementos de lo social. Punto de cruce donde, a partir de la objetivación del imaginario social instituido propio de una formación social dada, se cuajan los roles asignados (roles de hombre, de mujer, de madre, de padre, de trabajador, etc.); roles que están al servicio de mantener y reproducir ese orden social dado.

Este proceso conlleva grados importantes de consenso social y mantiene unida a una sociedad en el plano de la subjetividad colectiva. Implica interpretaciones colectivas solidificadas socialmente, que intentan clausurar todo intento de interpelación que trajese el riesgo de poner en cuestión las certidumbres sobre las que asienta 
su identidad esa formación social. Aún en procesos que dan cuenta de una formación social basada en la cooperación, la justicia y la igualdad, pueden perdurar, aunque de modo menos hegemónico, cristalizaciones efectivas de imaginario social correspondientes a formaciones sociales anteriores.

Los niveles de consenso facilitan la invisibilización o naturalización ${ }^{1}$ de los malestares, estableciéndolos no solo como normales, sino que sus pautas quedan sujetas a un grado de narcisización. Por ejemplo, cuando preguntamos "¿Qué es ser buena madre?", se suele responder con énfasis: "la que se desvive por los hijos", "la que ama sin medida", "la que lo da todo". Y sin embargo se puede pensar, desde lo saludable, que tener un hijo no es tener menos vida ("desvivirse"), no implica no poner límites ("sin medida"), ni tampoco un grado de abastecimiento absoluto ("darlo todo"). Pero, sin embargo ¿qué es lo que queda valorizado desde el imaginario social?

En aras de una sana autonomía nosotros decimos "todo lo que un niño/a puede tiene que hacerlo" y desde lo hegemónico se promueve "todo lo que quieren, pueden conseguirlo". Esto implica pautas de crianza consideradas como normales, pero que sin embargo, conllevan graves distorsiones para el proceso de crecer (dificultad con los desprendimientos, proceso de triangulación, ejercicio de autoridad, etc.).

Así observamos, que por un lado se espera que un niño/a de 6 años aprenda a escribir, lo cual tiene que ver con la comprensión de los códigos de la cultura para ir desarrollando autonomía y por otro lado se le da la comida en la boca, o se lo viste, o se lo baña, o se "le hacen" los deberes, etc. Se le pide responsabilidad a un púber y junto a ello se le recoge la ropa del cuarto, se le seca el baño, se le lava a veces la cabeza, se le imponen los gustos en la ropa, se le despierta por la mañana, etc.

Expresiones de las consecuencias de esta naturalización no saludable, la encontramos en los niveles de agobio y desgaste que se presentan en la crianza actual, expresados en la queja constante de los padres y madres acerca de "los niños nos desafían", "es que hoy vienen terribles", "hace lo que quiere con nosotros", "siempre tienen el mando" (de la TV), "me puede", "siempre se sale con la suya”, "se nos van de las manos", junto a la inhabilitación del lugar adulto en relación al ejercicio de una autoridad necesaria para ayudar a crecer.

En el caso de púberes cuando los muchachos y muchachas comienzan a interesarse de otro modo por el "afuera" e inician un nuevo desprendimiento, los papás y mamás suelen no comprender el sentido de las nuevas relaciones ("pierdes el tiempo en la calle", "te pasas tonteando en lugar de hacer lo que tienes que hacer que es estudiar”, esos amigos te distraen, ¿porqué tantas horas al teléfono? ¿tanto tienen que decirse? ....).

Observamos estas dependencias aún persistiendo en las relaciones intrafamiliares de adultos. Los padres y madres se siguen postulando como "padres-madres de crianza" de hijos mayores y se suele achacar como causa de las mismas a los tiempos de trabajo de los hijos, a las prisas y trajín diario, pero en muchos casos opera más la dificultad de entender la importancia de los espacios personales y sus necesidades y los límites de cada uno y de cada rol.

A pesar del alto nivel de consenso, estos malestares son generadores de importantes costos en SaludBienestar de la población. Es por esto, por lo que adquiere especial significación trabajar en la delimitación de su campo, y en la conceptualización teórica-metodológica para una adecuada intervención.

Plantearemos brevemente algunas consideraciones teóricas más, sobre la relación entre lo psíquico, lo social y los consensos establecidos.

Castoriadis (1983, p.181), señala que lo psíquico y lo social son radicalmente irreductibles el uno al otro, a la vez que absolutamente indisociables. Con el concepto clave de imaginario radical, con sus dos vertientes, el de imaginación radical que se expresa en y por el inconsciente, y el de imaginario social, que se expresa en y por la sociedad (lo histórico-social), introduce aportes fundamentales para la superación de dualismos en la

${ }^{1}$ Tello (2003) en su trabajo sobre Castoriadis señala: "El imaginario social instituido establece qué es un hombre y una mujer, qué es el estado, la libertad, la honestidad (...) qué es un niño, un delincuente, la moral, etc." (p.100). Citando a Adamson (2001) describe al imaginario social eficaz como aquello que compartimos, aquello que nos da certidumbre y que nos parece lógico y obvio, de sentido común, aquello que establece que "las cosas son asî". De este modo, cada cultura establece qué es lo percibible, lo pensable, lo significable (p.101).

Esto implica, siguiendo el pensamiento de Castoriadis, un grado de clausura, pero asimismo desde el concepto de imaginario radical y capacidad instituyente, cada sociedad lleva la potencialidad de su autoalteración. 
relación psique-sociedad. Este imaginario social que opera como organizador de sentido de los actos humanos y regula los comportamientos, construye realidad y tendrá una incidencia directa en el devenir psíquico. Desde aquí nos habla de la determinación que concierne a la materia misma del sujeto, que remite al mundo que lleva en él, haciendo "entrar la calle en lo que podría creerse su alcoba" (op. cit. p.179), concluyendo que "el sujeto efectivo por tanto, es aquel penetrado de parte a parte por el mundo y por los otros".

"Este autor que alza su universo conceptual alrededor de su innovadora teoría de la imaginación, dirá que es en lo imaginario donde una sociedad (op. cit, p.222) debe buscar el complemento necesario de su orden" (Cucco, 2006b, p.3).

E. Pichon Rivière nos dirá que a partir de la necesidad se comprende el carácter social de la esencia del sujeto. La cría humana "es un ser de necesidades que sólo se satisfacen socialmente en relaciones que lo determinan, por tanto no es un sujeto relacionado, es también un sujeto producido" (Zito Lema, 1985, p.107). Esto implica enfatizar el papel que juegan las relaciones sociales como posibilitantes del psiquismo, así la concepción de sujeto relacional del psicoanálisis deja paso al sujeto agente productor y producido en un sistema vincular (García \& Waisbrot, 1981, p.11).

La cría humana, atravesada entonces por la necesidad de supervivencia tiende a la búsqueda de satisfacción. Allí se encontrará con otro humano. Al ofrecer el pezón, ese otro humano está ofreciendo algo más que el pezón y la leche. Sobrepasa la satisfacción meramente somática ofreciendo un cuidado sexualizante en la doble función, en el decir de S. Bleichmar (2000, p.145), la función pulsante que inscribe la pulsión y la función ligadora responsable de la represión originaria, la fundación del inconsciente y los inicios de la formación yoica. De este modo la cría, esa entidad biológica con débiles montantes adaptativos, recibe la inscripción en la cultura, el sello de su humanización. Esto, en el sentido de su doble existencia (Freud, 1972) en tanto ser para sí mismo su propio fin y en tanto elementos de una cadena de la cual es servidor, sino contra su voluntad, en todo caso sin la intervención en ello.

Por tanto también es amamantado, acunado, mirado desde los adultos primordiales como representantes de un mandato social. Mandato que desde lo hegemónico, con la fuerza de lo instituido, como señalábamos, moldea hoy pautas de crianza que distorsionan gravemente organizadores básicos (narcisización, duelo, proceso identificatorio, límites, triangulación, etc.) responsables de la construcción psíquica.

La sociedad busca entonces, instituir interpretaciones dominantes que se arraiguen en las subjetividades, intentando clausurar, como ya señalábamos, todo intento de interrogación, dado que esto entraña el riesgo de cuestionar las certidumbres sobre las que se asienta su identidad. Esta institución de las significaciones instaura las condiciones de lo factible y mantiene unida a una sociedad, en el plano de la subjetividad colectiva. Así, toda formación económico-social "sujeta" su orden que, en este caso, es un orden al servicio de relaciones humanas mercantilizadas, propias de un orden social que se rige por la lógica de la obtención de beneficio y no por la lógica de la resolución de las necesidades de todos y todas. Orden que requiere para la convivencia social, sujetos individualistas y dependientes.

En la búsqueda de alternativas a los niveles de precarización subjetiva actual, nuestra contribución intenta enriquecer un campo de conocimientos: el de la intervención sobre los malestares de la vida cotidiana como espacio de transformación social en el marco de la Metodología de los Procesos Correctores Comunitarios (ProCC). Desde esta concepción rescatamos la vida cotidiana como un lugar privilegiado de estudio y trabajo. A pesar de ser considerada con cierto desdén como lugar de mera empiria, es imprescindible su estudio toda vez que se quiera comprender la interrelación entre el mundo económico-social y la vida humana con sus contradicciones. "La vida cotidiana se presenta como el lugar de producción y reproducción de los sujetos, y a través de ella se expresan los malestares que conforman el ámbito de la Normalidad Supuesta Salud" (Cucco, 2004, p.13)

Por otro lado entendemos el espacio grupal como matriz viva, lugar de génesis y neogénesis de la subjetividad, lugar diagnóstico y operativo por excelencia. Junto a la dimensión institucional, constituye el espacio de intermediación donde, a través de su dinámica, permite comprender como lo macro puede transmutarse en lo 
más íntimo de cada persona; dejar allí en la formación de la propia subjetividad la marca, la inscripción social, cultural e intergeneracional.

Consideramos el método grupal como espacio imprescindible para lograr independencia de los aspectos instituidos que son parte constituyente de nosotros mismos y para desarrollar capacidad instituyente para la construcción de contraconsensos. Cabe señalar que los consensos instituidos no desaparecen fácilmente y perduran en sus efectos a pesar incluso de los cambios en las condiciones sociales y materiales En este sentido se hace necesario contar con una metodología de trabajo grupal adecuada y específica.

Desde la Metodología ProCC, aportamos el método de Grupo Formativo como un dispositivo idóneo, que sin entrar en niveles terapéuticos propiamente dichos (Cucco, 2004, p.1).

"es un método grupal particularmente diseñado para abordar los malestares de la cotidianidad. Su finalidad específica apunta a conseguir grados de independencia de lo imaginario social instituido-enajenante, potenciando el desarrollo de protagonismo personal-social para operar transformaciones sociales. Esto naturalmente incidirá en los comportamientos concretos que materializan la vida cotidiana. Es decir, trabaja para la conformación del sujeto autónomo, para lo que es condición desarrollar la capacidad de reflexividad que implica la posibilidad de la puesta en cuestión de sí mismo. En esta puesta en cuestión, es importante que la interpelación vaya más allá de los interrogantes autorizados por el sistema de lo instituido enajenante”.

Conlleva entonces "desarropar" aquello que nos constituye, aguantar el vacío de romper nuestras certidumbres. Este trabajo implica una propuesta de intervención comunitaria a través de los Programas ProCC, donde con gran eficacia y sin tocar lo "más íntimo personal" se abordan cuestiones esenciales del malestar de las gentes y no se deriva a procesos psicoterapéuticos. Fomentar el protagonismo, necesario en el desarrollo de toda acción participativa para la construcción de alternativas, es objetivo fundamental.

Bleger (1983, p.181) decía que muchos "enfermos" más que sufrir desadaptación a la sociedad, sufren, por el contrario, el ser adaptados a las condiciones alienadas de la sociedad. Desde esta perspectiva nos preguntamos ¿cuánto del abordaje de estos malestares es tarea de la intervención grupal-comunitaria que proponemos?, ¿cuánto puede merecer una mirada clínica?, ¿en qué lugar quedan las acciones preventivas?. Pensamos que discriminar el objeto de estos niveles de intervención es esencial en el trabajo en salud. Considerar el ámbito de la Normalidad Supuesta Salud nos significó un punto de inflexión para ello permitiéndonos diferenciar el ámbito de los Procesos Correctores Comunitarios, el de la Atención Clínica y el de la Prevención. Por otro lado hemos desarrollado, en el trabajo con padres y madres dentro de la clínica de niños/as y adolescentes, lo que en el quehacer de la Clínica ProCC llamamos Entrevistas Clínico-Pedagógicas. Estas son pedagógicas dentro de un encuadre clínico y facilitan mucho el trabajo ya que permiten articular con los padres y madres un $\mathrm{ECRO}^{2}$ de trabajo inicial respecto a los aspectos naturalizados en la crianza, que si no son trabajados siguen operando interfiriendo el tratamiento.

\section{Escuela para padres y madres ¿una necesidad?}

Intentaremos caracterizar nuestra propuesta de intervención sobre la crianza, para que padres/madres, docentes y profesionales en general, puedan enriquecer su reflexión acerca de estos grandes retos que nos presenta el proceso de crecer actual. En este sentido tomaremos como ejemplo la temática de crecer, desarrollo de la autonomía y límites y la función del adulto. Rescataremos en primer término y desde el punto de vista teórico, el papel que cumplen los límites desde su función estructurante del psiquismo en el proceso de humanización.

\footnotetext{
${ }^{2}$ Esquema Conceptual Referencial y Operativo.
} 
Nos referiremos en primer lugar a un nivel teórico que hace a la comprensión del tema, que denominamos Teoría 1, que (Cucco, Córdova, \& Rebollar, 2010, p.214):

“incluye los referentes generales y teóricos particulares, asumidos crítica y creativamente que permiten definir un objeto de estudio y trabajo". Nos referiremos posteriormente a "un segundo nivel, el de "Teoría 2" que remite al cuerpo teórico que constituyen los Indicadores Diagnósticos de Población. La T1 define por ejemplo, ciertos referentes de "qué es crecer" y la T2 arma el cuerpo teórico de "cómo se crece hoy”. Esto implica un ajuste significativamente distinto entre lo teórico y lo empírico”.

Estas conceptualizaciones de T1 y T2 representan un punto clave a la hora de intervenir ya que permiten plantear el trabajo con padres y madres por ejemplo, desde el análisis crítico de las contradicciones cotidianas y evita el riesgo de asumir la posición del "deber ser". Dicha posición es la que se promueve desde lo hegemónico en las acciones profesionales, implica un acto de retórica autoritaria o de violencia simbólica -en términos de Aulagnier-, e impotentiza tanto a los profesionales como a la población.

Situando entonces para nuestro tema algunos elementos de T1 consideramos que, a lo largo de la espiral del crecimiento los límites van permitiendo operar el tránsito desde la indefensión y la dependencia a la adquisición de autonomía y hablamos por tanto, de "límites para que los niño/as crezcan, no para que obedezcan".

Partimos, como señalábamos más arriba, de lo irreductible e indisociable de lo social y el devenir subjetivo. Aulagnier (1977, p.32) de forma muy poética alude a ello cuando dice: "En el momento en que la boca encuentra el pecho, encuentra y traga un primer sorbo de mundo". A partir de la necesidad, dirá Pichon Rivière (1992, p.7), se comprende el carácter social de la esencia del sujeto que es emergente de un sistema vincular, donde el interjuego necesidad-satisfacción opera como causa interna de su desarrollo, siendo esta experiencia la base y fundamento de la subjetividad. Por tanto, la cría humana es un ser indefenso de necesidades que se satisfacen por un otro desde un vínculo siempre social. La mamá mira, acaricia, habla a su bebé y está ofreciendo algo más que el pezón y la leche. Su cuidado va más allá de la satisfacción meramente somática, iniciándose un proceso donde ese otro se brinda para formar lo más íntimo del sujeto, iniciándose, desde la fusión, un camino de separación.

Es esencial el papel que en los vínculos tempranos ocupa este proceso de fusión-separación y lo transicional, ya que permite el pasaje de un estado de indefensión-dependencia, a un estado creciente de autonomía, que va conformando la capacidad de representarse como separado y diferenciado. El niño/a va adquiriendo, en este proceso, la noción de realidad como espacio exterior no reductible al propio, encontrando a su vez el yo su unidad y su límite. En este sentido definimos el proceso de crecer (Cucco, 1986, p.9) "como un camino de sucesivos desprendimientos hacia la autonomía desde cada nueva capacidad adquirida”.

Winnicott (1982, p.17 y ss) de forma sugerente señala que ante la indefensión del bebé que experimenta necesidades vitales, es importante que la madre responda ofreciendo el pezón en el justo momento en que éste lo necesita. Esto crea en el niño/a un sentimiento de poder y de omnipotencia con la ilusión de crear el mundo de su alrededor ajustado a sus necesidades. "Adaptación casi exacta, dice Winnicott (1993, p.147), a la necesidad, lo que le proporciona al bebé la ilusión de haber creado el objeto exterior". Paulatinamente la madre abandona esa primera actitud eficiente, prometedora de ilusión de omnipotencia, y desde una actitud de "madre suficientemente buena", va desarmando esa omnipotencia con moderadas desilusiones, en consonancia con la creciente capacidad del bebé para encarar ese proceso. Esto no se logrará si antes no le ofreció suficientes oportunidades de ilusión; ya que esto es lo que le habrá permitido, con el paso del tiempo, "sentirse confiado en que habrá de encontrar el objeto de su deseo, lo cual significa que va tolerando gradualmente la ausencia del objeto" (op. cit., p.152); así se inicia en el concepto de realidad externa, donde los objetos aparecen y desaparecen, se instala la capacidad de espera y la resistencia a la frustración. Winnicott llamó "capacidad de estar solo" no a una soledad defensiva, sino a la de un yo fortalecido que introyectó el objeto. Esta capacidad de estar solo permite entender los vínculos entre la cohesión del yo y las relaciones con el otro. "El sujeto puede estar físi- 
camente solo, pero no psíquicamente abandonado" (Hornstein, 2000, p.58). Esta desilusión, dice este autor, sigue siendo una de las tareas inapelables de padres-madres y educadores. Si dejamos al niño/a en la vivencia de la "exacta adaptación", ésta se parece a la magia, lo que le impedirá el crecimiento.

Lo dicho anteriormente implica un proceso en donde se transforma el deseo hacia el objeto, en investimiento yoico, a través de los procesos de identificación y duelo que le permiten al niño conservar aquello que el principio de realidad le obliga a abandonar (la desilusión de la omnipotencia, en sentido winnicottiano).

Para que este yo pueda devenir se pone en juego el narcisismo y la constelación edípica de los adultos. Es condición un narcisismo con capacidad estructurante de las figuras primordiales, capaz de soportar los grados de indefensión del bebé y su ser desintegrado, y capaz a su vez de ir generando las sucesivas y adecuadas separaciones de acuerdo a los grados crecientes de desarrollo del niño. Es decir, que el adecuado interjuego entre los momentos de fusión y separación, dependerán de la acción del otro primordial, que a través de los límites adecuados, facilitará o no su función estructurante para la formación del incipiente aparato psíquico en el niño/a. Esto remite a uno de los más bellos actos de amor del ser humano, a través del cual, asumiendo también el adulto el duelo de desilusionar al bebé de su omnipotencia, renunciando a ella, es capaz de entregarle su posibilidad de crear el espacio de lo representacional, espacio simbólico que hace a lo específico de ser sujeto humano. Aspectos éticos y estéticos se funden aquí para poder otorgar al otro su capacidad de "despegar" en la vida. Intersubjetividad en la que convergen los mayores niveles de compromiso vincular, junto a las mayores posibilidades de individuación para ambas partes del vínculo.

Para que estos "desprendimientos" sean saludables es necesario asegurar el mantenimiento de cierta constancia en la relación con los adultos referentes, constancia y movilidad a su vez desde que el vínculo se va reacomodando permanentemente en función del desarrollo de la creciente autonomía del niño/a.

Se hace necesario también el saber identificar los momentos de "prendimiento-desprendimiento", así como que es a través de los límites y del ejercicio de la autoridad como se opera la separación y la delimitación de los nuevos "lugares". Concebimos la autoridad como aquella función que gestiona el lugar de cada uno en función de las necesidades de ambas partes del vínculo.

El proceso descripto anteriormente implica por tanto que, desde un buen "holding" se comprendan las necesidades y los ritmos propios del bebé (niño/a, púber, etc.), y con capacidad de "reverie" se posibilite la metabolización de los niveles de ansiedad, facilitando desde los duelos pertinentes, el acceso a mayores niveles de integración. Será importante no hacer de más y sí lo que necesita, acompañando en aquello que puede desarrollar con ayuda del semejante (Zona de Desarrollo Potencial en sentido vigotzkyano). Desde aquí la separación debe ser sostenida con firmeza, sin dar lugar a la esperanza, ya que si "el chupete se lo comió el gato", el niño entenderá que no lo tiene por algo accidental y no porque se acabó el tiempo del chupete.

Y así, podríamos seguir desarrollando la especificidad de cada etapa en este camino de las sucesivas separaciones para lograr autonomía, hasta el final de la adolescencia.

Sin dejar de resaltar la complejidad de lo brevemente descripto, que hace referencia a la construcción del sujeto psíquico autónomo y saludable, las grandes dificultades que observamos hoy en la crianza no responden tanto a esa complejidad, como al desconocimiento y precarización masiva de estos procesos (que sistematizamos a través de los Indicadores Diagnósticos de Población).

En el caso del embarazo y desde el punto de vista biológico, está claro cuál es el tiempo de estar "prendido" y de favorecer ese "prendimiento", so pena de riesgo fatal frente a un inadecuado adelanto o retraso; y cuál es el momento del "desprendimiento" y del parto. Pero con el bebé en brazos, y de allí en adelante, mientras dura el proceso de crianza, o sea, hasta finalizar la adolescencia, ¿están igual de claros los momentos de "prendimiento” y de los “desprendimientos”? ¿Está clara la necesidad y función que cumplen los límites? ¿Está clara la función del adulto?

Desde nuestra praxis observamos en primer lugar un grado importante de desconocimiento de estos procesos que deberían constituir un saber básico y necesario de la población en general. Y por otro lado, indicadores actuales como la fragilidad narcisista y la inhabilidad en el sostenimiento de un vínculo, el cortoplacismo, 
la inmediatez y la descualificación de lo procesual, el vacío de normas y la futilidad del esfuerzo, la pérdida de sentido, etc., no garantizan que los adultos primordiales sean capaces de reacomodar permanentemente su lugar, acorde a los avances de la creciente autonomía del niño/a y haciendo un buen ejercicio de la autoridad. Más bien hoy muchos adultos actúan desde la sobreprotección y desde las promesas de abastecimiento absoluto y abdican de su lugar y los niño/as "todo lo que quieren pueden conseguirlo", desajustando sus lugares, en lugar de "todo lo que el niñola puede, tiene que hacerlo".

\section{El "qué hacer"}

Nuestra propuesta de intervención comunitaria, a través del Programa ProCC de Escuela para padres y madres trabaja temáticas como la ejemplificada, relacionada con el papel que juegan los límites en el desarrollo de la autonomía, así como las concernientes a la problemática del grupo familiar, los roles masculino y femeninos y su carga socio-cultural, las etapas evolutivas y los micromecanismos distorsionados del crecer actual, la función del ser padres y madres y los retos actuales, entre otras. Crea espacios de reflexión sobre estas problemáticas desde un encuadre de proceso corrector, que genera independencia de lo instituido y que se diferencia de las acciones terapéuticas y preventivas. Brinda elementos de análisis para posibilitar transformaciones de los micro procesos de la crianza puestos en juego en el contexto de la vida cotidiana actual. Fomenta el desarrollo del protagonismo personal-social para la búsqueda de alternativas a las situaciones planteadas.

Está dirigida a población general, incluyendo por supuesto las problemáticas de niveles más precarizados, pero sin plantear que es una intervención solo dirigida a ellos, habida cuenta que el concepto de malestar que se plantea hace referencia a aspectos estructurales que afectan y distorsionan la crianza en general.

Trabaja con el método de Grupo Formativo. Esta metodología centra el aprendizaje cuidando la especial relación entre los aspectos temáticos y dinámicos del proceso grupal. Es activa y participativa y se utilizan diversos recursos según el tipo de programa, los objetivos propuestos y las características de los participantes. Está a cargo de profesionales especializados.

El diseño de los programas implica un análisis riguroso de la necesidad desde los Indicadores Diagnósticos de Población y la articulación de una estrategia de intervención considerando la relación entre necesidad y demanda. A partir de esto se considera el encuadre más operativo a implementar, según recursos y posibilidades.

Cada programa cuenta con sus objetivos, contenidos y metodología y se desarrolla a partir de un número de reuniones de diversas modalidades. En la programación, desde un hilo conductor, se organiza la secuencia de las temáticas de las reuniones. Este responde a un hilo lógico, propio de los requerimientos del tema y a un hilo elaborativo, propio de los requerimientos de los Indicadores Diagnósticos de Población. Esta organización de los temas facilita los procesos elaborativos y la resolución de los obstáculos propios de un proceso de reflexión para la transformación.

Cada reunión a su vez consta de objetivos, contenidos y metodología, así como de la sugerencia del recurso didáctico más adecuado según la problemática a trabajar.

Entre los recursos utilizados cuenta de modo particular el uso del Juego Dramático que es una aplicación original de la dramática dentro del método de Grupo Formativo. De este modo lo que se lleva a escena, según los temas, es el perfil de un Indicador Diagnóstico de Población sin necesidad de entrar en relatos personales. Por ejemplo, situaciones cotidianas significativas de algún modo vividas por todos y que son expresión de distorsiones en las pautas de crianza que conllevan malestar y preocupación. La identificación con las mismas, con la distancia a su vez de no ser personales, facilita un importante nivel de reflexión. A partir de allí se brindan elementos de análisis que permiten la construcción de criterios. Se fomenta así el desarrollo personal y social para resolver los conflictos planteados. 
La construcción de los Programas ProCC conlleva una gran arquitectura tanto teórica como metodológica de mucha minuciosidad, pero asume ante los participantes un nivel ameno y asequible en la presentación de los conceptos. La idea es partir de los hechos del día a día en el ser padres y madres, del día a día de la realidad familiar y brindar un espacio grupal que permita un trabajo de análisis sistemático y operativo, desarrollando el placer de la construcción colectiva.

Los resultados de nuestra praxis en este modelo de Escuela para padres y madres, con más de 30 años de desarrollo y realizada en diferentes espacios nacionales e internacionales, nos ha llenado de satisfacciones por la calidad y eficacia de los logros. Expresiones como “Cursos como este nos humanizan", nos alienta a seguir trabajando desde esta concepción, por una vida cotidiana más humana, más solidaria, más saludable, desarrollando el protagonismo y la acción participativa para la solución de las problemáticas planteadas.

\section{A modo de conclusión}

Teniendo en cuenta que hoy observamos: una subjetividad en riesgo; una precarización de los espacios de construcción simbólica inherentes a lo que es la humanización; la distorsión, desde una fina orfebrería, de pautas de crianza saludables, junto a la "extinción" de la posición de adulto; consideramos urgente y priorizado promover un trabajo grupal y comunitario en este sentido.

\section{Referencias}

Adamson, G. (2001). La psicología social frente al $3^{\circ}$ milenio. Buenos Aires: Editorial Labriego.

Aulagnier, P. (1977). La violencia de la interpretación. Buenos Aires: Amorrortu.

Bleichmar, S. (2000). Clínica psicoanalítica y neogénesis. Buenos Aires: Amorrortu.

Bleger, J. (1983). La psicología de la conducta. Buenos Aires: Paidós.

Castoriadis, C. (1983). La institución imaginaria de la sociedad. Vol I Barcelona: Tusquets.

Cucco, M. (1986). Un enfoque, una línea de trabajo que intenta penetrar en la comprensión de nuestro ser social. Ponencia. Trabajo presentado en Seminario: Las bases psico-sociales del comportamiento agresivo, Fundación Pablo Iglesias, Madrid.

Cucco, M. (2003). Algunos puntos de partida y tres organizadores básicos de la subjetividad. En M. A. Rebollar. Intervención Comunitaria, (p.81). La Habana: Cenesex.

Cucco, M. (2004). El Grupo Formativo. Sus principios metodológicos. Trabajo presentado en I Taller Nacional de Coordinadores de Grupo Formativo. La Habana. Cuba.

Cucco, M. (2006a). ProCC: Una propuesta de intervención sobre los malestares de la vida cotidiana. Buenos Aires: Atuel.

Cucco, M. (2006b). Malestares cotidianos y micromecanismos subyacentes. Los IDP como concepto clave para su comprensión. Trabajo presentado en II Taller Nacional de Coordinadores de Grupo Formativo. La Habana.

Cucco, M. (2009). Del desatino social a la precariedad narcisista. Trabajo presentado en XIII Congreso Argentino de Psicología. "Compromiso social frente a los problemas actuales". Córdoba-Argentina.

Cucco, M., Córdova, M.D. y Rebollar, M. (2010). La intervención sobre los malestares de la vida cotidiana. Madrid: Nuevos escritores.

Freud, S. (1972). Introducción al narcisismo. Madrid: Biblioteca Nueva.

Garcia, M. y Waisbrot, D. (1981). Una vuelta de espiral dialéctica. Argentina: Centro Editor Argentino.

Hornstein, L. (2000). Narcisismo. Buenos Aires: Paidós.

Pichon Rivière, E. (1992). Teoría del vínculo. Buenos Aires: Nueva Visión.

Tello, N. (2003). Cornelius Castoriadis y el imaginario radical. Madrid: Campo de Ideas. S.L. 
Winnicott, D. (1982). Realidad y juego. Barcelona: Gedisa.

Winnicott, D. (1993). La naturaleza humana. Buenos Aires: Paidós.

Zito Lema, V. (1985). Conversaciones con Enrique Pichon Rivière sobre el arte y la locura. Buenos Aires: Cinco.

Manuscrito recibido: 31/07/2012

Revisión recibida: 02/10/2012

Manuscrito aceptado: 03/10/2012 